\title{
APLANANDO LA CURVA DEL CONSUMO ELÉCTRICO CON FOTOVOLTAICOS Y HÁBITOS AHORRADORES EN UN DEPARTAMENTO EN LIMA (2017-2020) ${ }^{1}$
}

\section{FLATTENING THE CURVE OF ELECTRIC CONSUMPTION WITH PHOTOVOLTAICS AND SAVING HABITS IN AN APARTMENT IN LIMA (2017-2020)}

D0I: https://doi.org/10.47796/ra.2021i20.551

RICHARD HENRY VALDIVIA-SISNIEGAS ${ }^{2}$

Investigador independiente

https://orcid.org/0000-0003-0783-6831

sunarch.eirl@gmail.com

RESUMEN

Con el confinamiento por el Covid-19, el consumo eléctrico aumentó para numerosas familias en Perú, así como las tarifas eléctricas. El objetivo de esta experiencia fue demostrar la posibilidad de instalaciones fotovoltaicas, incluso en condiciones no ideales, además de estudiar el consumo eléctrico integrando gradualmente aparatos eficientes y hábitos de ahorro. Se muestra el monitoreo de 4 años (2017-2020) de un departamento en Lima, con diversos sistemas fotovoltaicos de aporte parcial instalados en la fachada mediante un soporte patentado, para luego registrar y comparar la evolución de los consumos mensuales y anuales utilizando los recibos de electricidad. Finalmente, se calcularon ahorros de emisiones y retornos económicos. Los resultados indican ahorros considerables incluso durante la "nueva normalidad", por lo que son oportunos para la adaptación y mitigación al cambio climático, teniendo como eje central el cambio de hábitos de consumo de electricidad combinados con energía solar fotovoltaica.

\section{ABSTRACT}

With the lockdown imposed by Covid-19 pandemic, electricity consumption has increased for many families in Peru, as well electricity tariffs. The objective of this experience was to demonstrate the possibility of photovoltaic installations in apartments even under non-ideal conditions, in addition, to study the electricity consumption integrating gradually efficient appliances and saving habits. The article explains the monitoring of 4 years (2017-2020) in an apartment in Lima, with various photovoltaic systems for partial contribution incorporated in the façade by mean of a patented hanger, to register and compare the evolution of monthly and annual consumption using electricity billing receipts. Finally, the savings in emissions and economic returns have been calculated. The results show a considerable level of savings in electricity and even more during the "new normal", being an opportunity for the adaptation and mitigation to climate change, and considering the change of habits in electricity consumption combined with photovoltaic energy as central axis.

\footnotetext{
Investigación independiente realizada con el financiamiento de la empresa SUNARCHE.I.R.L. dedicada a proyectos y consultorías sobre arquitectura, eficiencia energética y desarrollo sostenible.

Arquitecto por la Universidad Ricardo Palma (Lima - Perú) con Maestría en Estudios Avanzados en Arquitectura y Desarrollo Sostenible (EPFL, Suiza y UC Lovain-La Neuve, Bélgica). Gerente General de SUNARCHE.I.R.L. Arquitecto proyectista, docente universitario, investigador y consultor del Foro Ciudades para la Vida.
} 
PALABRAS CLAVE: adaptación y mitigación, eficiencia energética, energía fotovoltaica, consumo eléctrico, Covid-19.

\section{INTRODUCCIÓN}

Lima experimentó un fuerte desarrollo inmobiliario del 2005 al 2015 -el cual continúa, pero en menor intensidad-, y los departamentos son ahora una cantidad importante de viviendas. En 2015, se publicó la Ley de Generación Distribuida ${ }^{3}$, que debió publicar su reglamento seis meses después. Sin embargo, recién en 2018 se prepublicó un proyecto de reglamento ${ }^{4}$, de modo que su aprobación quedó pendiente de manera indefinida. Existen todavía entrampamientos legales, económicos y técnicos para la generación distribuida (Ramos, 2020), (Matos Ortega y Vargas Guevara, 2019), (Sciutto, 2019), sin netmetering ${ }^{5}$ o netbillling ${ }^{6}$ y sus beneficios. Por ello, aún no existe un clima favorable para instalaciones solares domiciliarias, a lo cual se suma la creencia de que en Lima lo solar no funciona, desconociendo el potencial de la radiación solary sus oportunidades.

De otro lado, en el modelo eléctrico peruano basado en un mercado mayorista de electricidad (Okumura, 2015), los costos de las tarifas dependen de la coyuntura y del comportamiento en el mercado de los diversos actores en la cadena de generación, transmisión y distribución. La regulación laxa ha llevado, según expertos, a crear distorsiones en el sistema de precios (Gutiérrez, 2019), elevando
KEYWORDS: adaptation and mitigation, energy efficiency, photovoltaic energy, electricity consumption, Covid-19.

el costo de las tarifas para consumidores regulados (tarifas domiciliarias) y reduciendo las tarifas de consumidores libres (grandes mineras e industrias). Asimismo, las tarifas de electricidad se incrementan de manera imperceptible en el tiempo, lo cual es necesario visibilizar para deducir posibles ahorros en mediano y largo plazo. Actualmente, la tarifa residencial de Lima es la segunda más cara en Sudamérica (Coronado, 2020), y la pandemia del COVID-19, que desde marzo de 2020 ha confinado a las personas en casa, ha demandado un incremento en el consumo de electricidad residencial de hasta el 50\% más de lo habitual en algunas viviendas, y hasta un $10 \%$ en las tarifas para los usuarios regulados ("Tarifa", 2021).

La Agencia Internacional de la Energía (EIA) propone como un indicador clave el consumo de energía por vivienda para el sector residencial (EIA, 2016, p. 47). El consumo de electricidad en viviendas es variable en el tiempo y depende de muchos factores (personas, clima, hábitos, instalaciones, etc.) En 2017 a nivel de cifras nacionales, el Organismo Supervisor de la Inversión en Energía y Minería (Osinergmin) remarcó la imperiosa necesidad de inculcar la cultura de ahorro en la ciudadanía peruana. Según este informe, la intensa campaña de ahorro de energía en el sector residencial en el periodo 1995-2001 logró

\footnotetext{
Decreto Legislativo No. 1221, del 24 de setiembre del 2015.

Resolución Ministerial No. 292-2018-MEN/DM, publicada el 2 de agosto del 2018

NetMetering (Medición Neta, conocido como crédito eléctrico): la energía solar que el cliente genera por sí misma se mide, de modo que cualquier exceso de electricidad que genere puede ser depositado o acumulado (crédito a favor) a la cuenta del cliente para un consumo futuro (Arias, 2017).

NetBilling (Facturación Neta): permite a los clientes solares generar electricidad para uso personal y vender cualquier excedente de energía a la compañía de servicios al por mayor o "tarifa de venta" (Arias, 2017).
} 
reducir el consumo eléctrico residencial en más del 10\%. Sin embargo, este se incrementó luego de 2005 hasta 2010, y a pesar de las campañas de ahorro en el 2008 y 2009 no se apreció una reducción, y permanece en un rango promedio de $122 \mathrm{kWh} /$ mes. El aumento coincide con el crecimiento económico y el boom inmobiliario experimentado en Perú (Ministerio de Energía y Minas [MEM], 2017). Aún se carece de investigaciones específicas o estudios de caso más detallados por vivienda.

Por otro lado, en Perú el uso de paneles fotovoltaicos es aún escaso y poco monitoreado. Un estudio en Lima sobre generación distribuida (Palacios et. al, 2020) indica que si existiera retribuciones como el netmettering, el retorno para estos sistemas sería de 6.9 años, mientras que con el sistema netbilling sería de 10.6 años, con áreas fotovoltaicas considerables: $9.74 \mathrm{~m}^{2}$ y $51.95 \mathrm{~m}^{2}$, situadas en techos, una condición no usual en departamentos.

Asimismo, la EIA define a la medición como el método más exacto para complementar las encuestas residenciales existentes y enriquecer a los modelos con datos sobre el comportamiento y consumo energético de los ocupantes. Sin embargo, su limitación radica en el costo de equipos de medición. Para superar esta limitación, se puede monitorear desde el medidor existente que es constantemente revisado por la compañía eléctrica. Se debe considerar el 'período del monitoreo familiar' ya que, los períodos de seguimiento pueden variar ampliamente, desde un medio día hasta seis años de control continuo. En consecuencia, la duración de un monitoreo también puede oscilar entre unos pocos meses hasta algunos años (EIA, 2016, p. 66).
Esta investigación trata del monitoreo del consumo eléctrico mensual/anual de un departamento, visualizando el desempeño histórico, fundamentalmente después de la instalación fotovoltaica y las medidas de ahorro energético (2017-2020). El objetivo fue monitorear el consumo de electricidad de un departamento en condiciones poco ideales para lo fotovoltaico, considerándolo como apoyo parcial, tanto en el balcón como en lugares próximos a las ventanas, en combinación con equipos y hábitos de consumo ahorradores que aprovechen condiciones naturales manteniendo condiciones confortables de vida. Se utilizó dicha información para efectuar balances de emisiones y ahorros que permitan evidenciar los aportes de esta interacción (reducción de emisiones) y ahorros económicos (recuperación de la inversión).

\section{MATERIALESY MÉTODOS}

\section{El Consumo en Departamentos en Lima}

Según el primer Reporte Nacional de Indicadores Urbanos (Periferia y World Wild Fundation, 2018) el promedio nacional de alto consumo de energía eléctrica en una vivienda a 2018 era de $228.11 \mathrm{kWh} / \mathrm{mes}$ (Lima), mientras que el promedio nacional de bajo consumo residencial es $44.37 \mathrm{~kW} / \mathrm{mes}$. Además, el consumo promedio en viviendas de Lima sería de $110 . \mathrm{kWh} / \mathrm{mes}$, consumo muy próximo a ser beneficiario del subsidio $\mathrm{FOSE}^{7}$. De otro lado, un sondeo a viviendas de los estudiantes de diversas universidades durante los años 20112014 (Valdivia-Sisniegas, 2015) en 183 departamentos informa que el consumo máximo en departamentos puede llegar a ser de $485 \mathrm{kWh} / \mathrm{mes}$, mientras que el promedio fue

FOSE (Fondo Social de Compensación Eléctrica) es un esquema de subsidios y recargos cruzados entre los usuarios de electricidad en base a sus consumos. Otorga una reducción tarifaria para zonas urbanas de $7.5 \mathrm{kWh} / \mathrm{mes}$ en la tarifa eléctrica a viviendas que consumen entre $100 \mathrm{~kW} / \mathrm{mes}$ y $30 \mathrm{~kW} / \mathrm{mes}$; y una reducción de $15 \mathrm{kWh} / \mathrm{mes}$ en la tarifa eléctrica a viviendas que consumen menos de $30 \mathrm{~kW} / \mathrm{mes}$ (Ley 27510, Ley que crea el Fondo de Compensación Social Eléctrica). 
$229.98 \mathrm{kWh} / \mathrm{mes}$ y el consumo más bajo fue $52.67 \mathrm{kWh} / \mathrm{mes}$.

En el departamento estudiado, el consumo de los 3 ocupantes registra un consumo variable de acuerdo al grado de ocupación y hábitos de consumo hasta el 2016, mientras que el promedio de los dos años anteriores a la instalación (2015-2016) fue de $208.60 \mathrm{kWH} / \mathrm{mes}$, consumo cercano al promedio alto de consumo en viviendas a nivel nacional.

\section{Condiciones de Radiación en Lima}

Lima se encuentra a 12.040 latitud sur y 77.02 longitud oeste. Cerca de la línea ecuatorial, la mayor parte de la radiación se recibe desde la parte superior de la bóveda celeste (cenit). El comportamiento de la radiación es variable en el año: mayor a finales de primavera y durante el verano e inicios del otoño, mientras que se reduce prácticamente a la mitad durante finales de otoño, todo el invierno e inicios de primavera, como se aprecia en los cálculos elaborados a partir de tablas de información solar disponibles (Censolar, 1993). La irradiación sobre superficies dependerá de la inclinación, orientación y posición de la superficie (Figura 1).A nivel específico, los cálculos (Figura 2) revelan que la mejor condición de irradiación está en las azoteas o techos (100\%), y es la superficie semihorizontal orientada al norte la más recomendada (99.21\% de irradiación anual). En el peor caso, la superficie semihorizontal orientada hacia el sur tampoco significa una pérdida considerable (98.04\% del total anual).

En multifamiliares existentes, resulta difícil usar las azoteas porque no todos los propietarios cuentan con derechos (propiedad de los aires o techos sin uso exigido por las reglamentaciones convencionales), así como la ubicación de instalaciones (ductos, cuartos de máquinas, tanques elevados, etc.) que generan sombras.
Figura 1. Radiación Solar Anual en Lima de acuerdo a la Orientación y Posición de Superficies W/m²

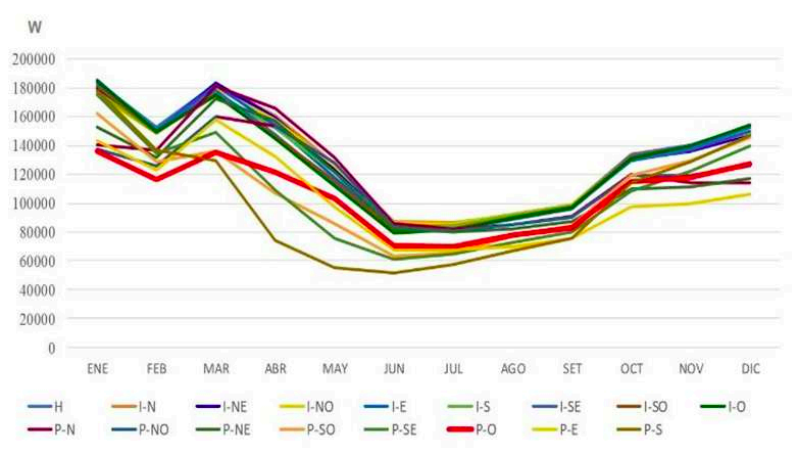

Nota: Se ha considerado $1 \mathrm{~m}^{2}$ de superficie horizontal $(H)$, inclinada a cielo abierto y orientada hacia 8 direcciones (l-...), e inclinada hacia cielo abierto adosada a pared orientada hacia 8 direcciones (P-...). La línea roja indica la opción disponible y utilizada para la instalación en el departamento (inclinada a cielo abierto y orientada al oeste: P-0). Elaboración propia en base a H-World Database.

Figura 2. Porcentaje Anual de Radiación Incidente en $1 \mathrm{~m}^{2}$ Horizontal y Bandejas Inclinadas con Diversas Orientaciones en Lima

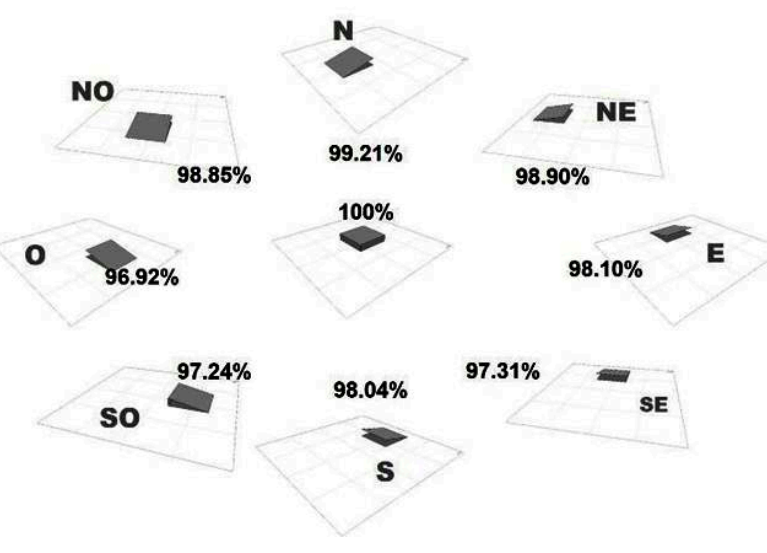

De esta manera, pensando solo en obtener una máxima ganancia solar en las azoteas y techos, no sería posible instalar sistemas fotovoltaicos en edificios ya construidos; lo cual haría imposible, en teoría, un escenario de conversión a favor de una adaptación y mitigación al cambio climático.

No obstante, la irradiación en superficies no ubicadas en azoteas, sino en bandejas semihorizontales en las fachadas, tendría una pérdida no mayor al $25 \%$ (bandeja 
semihorizontal situada hacia el sur con un $76.53 \%$ de irradiación anual) en el peor de los casos. Se considera que la mayor captación de radiación sería siempre de la parte superior (bandeja horizontal inclinada) pero con una obstrucción vertical en la parte posterior (pared) que sería la fachada del edificio (Figura 3).

Figura 3. Porcentaje Anual de Radiación Incidente en $1 \mathrm{~m}^{2}$ de Bandejas Semihorizontales Instaladas en Fachadas en Lima

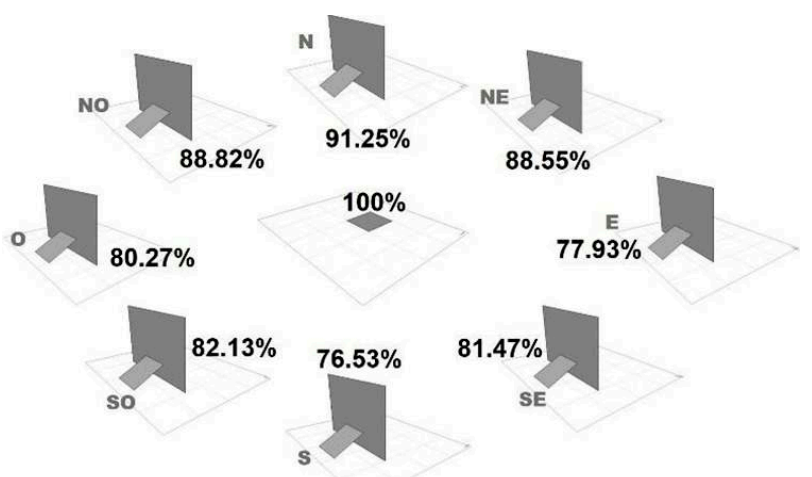

El departamento se ubica en el piso 11 de un edificio de 18 pisos, orientado hacia el oeste, por lo que no dispone de acceso a una azotea ni una orientación ideal -con una bandeja horizontal podría capturar solo el $80.27 \%$ de la radiación total anual en el mejor de los casos. El lugar con mayor disponibilidad para este tipo de departamentos es el balcón, ya que ofrece las condiciones óptimas para exposición a la radiación (Valdivia-Sisniegas, 2017), aunque como veremos, para el uso de sistemas pequeños se pueden instalar en ventanas.

\section{METODOLOGÍA}

Se trata de una investigación aplicada enfocada en el consumo y rendimientos, con carácter exploratorio de temas poco estudiados a nivel local como los sistemas fotovoltaicos y consumos de electricidad en departamentos. Se recopiló datos cuantitativos de manera cuasi experimental al no tener un control total de las variables. Estos datos se analizaron de manera longitudinal durante el periodo de tiempo mencionado. De acuerdo a la Figura 4, basados en el medidor eléctrico, se recopilaron los recibos de electricidad para generar un historial de los consumos ( $\mathrm{kW} / \mathrm{h} / \mathrm{mes}$ ) y costos de la electricidad (S/) desde 2017 hasta 2020. Asimismo, se pudo comparar con el historial previo de consumo del departamento, del cual se dispone información desde 2008. Se implementó un metro cuadrado de panel fotovoltaico de tipo mixto (microinversor + acumulación + 3 lámparas solares) teniendo en cuenta la disponibilidad de espacio y el aprovechamiento de las instalaciones eléctricas existentes. Por decisión propia del usuario, se fueron implementando mejoras de la eficiencia energética tanto en el cambio de ciertos artefactos, así como en el cambio de los hábitos de consumo. Se monitoreó los consumos a partir de la instalación y las mejoras de eficiencia energética implementadas. Se visualizaron los desempeños del consumo eléctrico y los costos en el tiempo mediante gráficos. Luego, se calcularon los ahorros energéticos y se tradujo en emisiones reducidas, así como los ahorros económicos, tomando en cuenta los incrementos en las tarifas eléctricas en los años de monitoreo y usando esta tendencia para una proyección anual de ahorros en años posteriores.

Figura 4. Esquema Metodológico

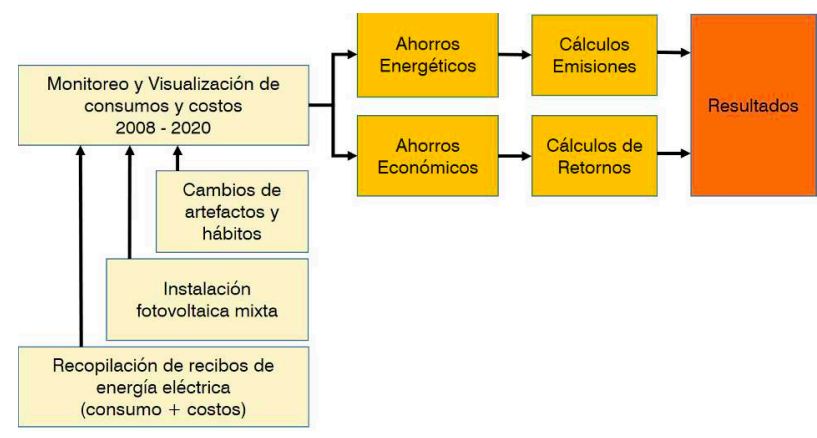

\section{DESARROLLO}

\section{Criterios de Diseño de la Instalación Mixta}

Para la instalación de $1 \mathrm{~m}^{2}$ de panel, se consideró suspender los paneles desde el techo 
del balcón, con el fin de no afectar la visual del balcón y mantenerse dentro de la propiedad horizontal del departamento, así como para servir de protección solar en días muy soleados. Las dimensiones del balcón (largo, ancho y alto) fueron determinantes para la selección de paneles. De acuerdo al modelamiento, una parte de la instalación tendría obstrucción solar parcial (Figura 5).

Se diseñó y patentó un sistema de montaje individual por panel. El dispositivo, denominado "soporte para paneles fotovoltaicos en balcones" (Perú Patent No. 002030-2018/DIN, 2018), permite el mantenimiento de limpieza y fue pensado para lugares como Lima, ya que las condiciones de humedad, lloviznas de invierno y la contaminación crean suciedad en superficies s e mihorizontales, reduciendo considerablemente la eficiencia de los paneles.

Figura 5. Modelo Tridimensional de los Paneles Suspendidos sobre el Balcón Seleccionado

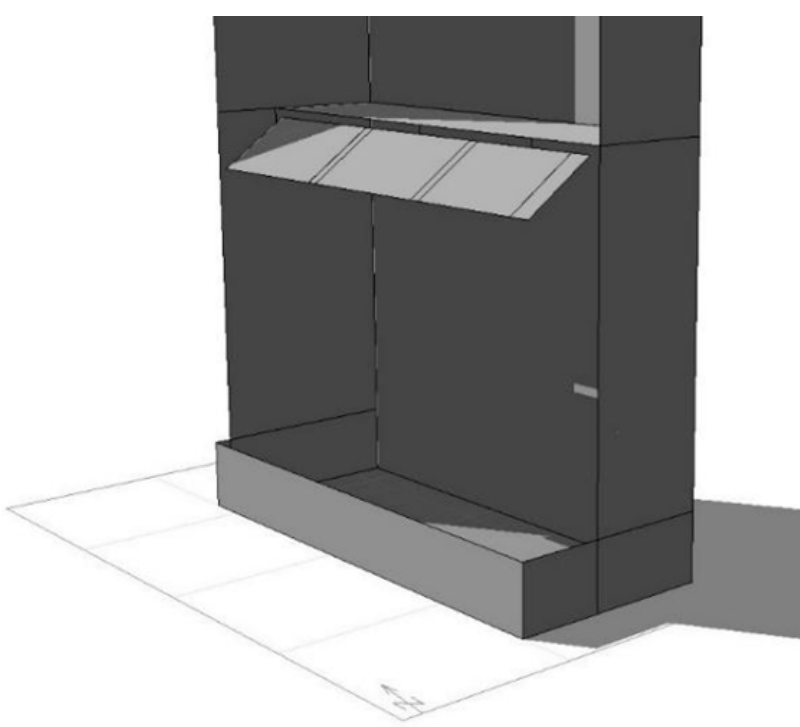

El mecanismo flexible del soporte permite la limpieza de los paneles desde el interior del balcón en condiciones seguras (Figura 5). Con este método de suspensión se pueden instalar en balcones de $2.80 \mathrm{~m}$ hasta $240 \mathrm{Wp}$. Este sistema ha llevado en el 2020 a una segunda patente con mejoras técnicas (Perú
Patent No. Exp: 001910-2020/DIN, 2020), y logró instalar hasta $320 \mathrm{Wp}$ en un balcón de características similares.

Figura 6. Paneles Fotovoltaicos en el Balcón Utilizando el Modelo de Utilidad: Soporte Colgante para Paneles Fotovoltaicos en Balcones (Perú Patent No. 002030-2018/DIN, 2018)

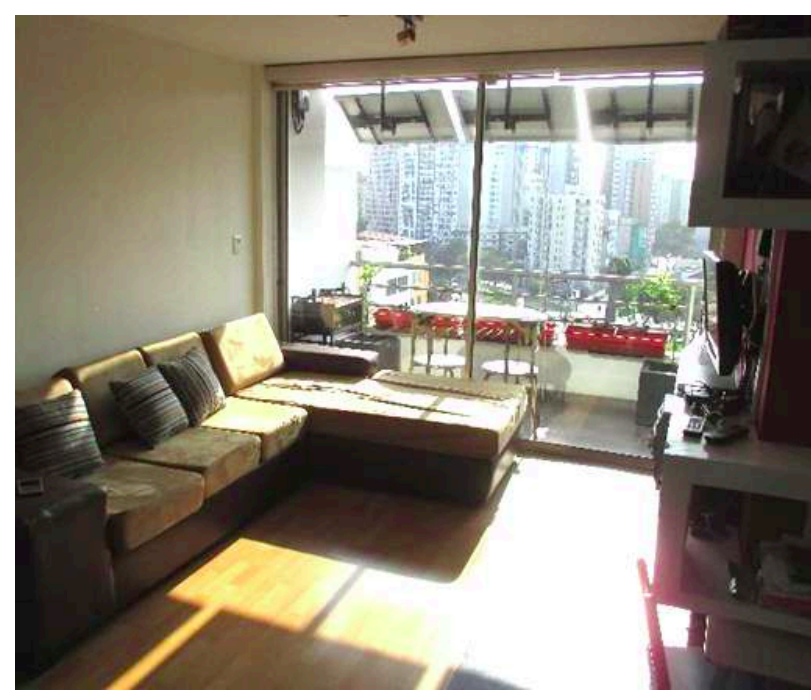

Fuente: Fotografía de Valdivia, R. (2018)

El sistema permite efectuar la limpieza desde el interior del balcón evitando riesgos de caídas a mucha altura. Debido a la ubicación de los paneles y la forma del edificio, uno de los paneles recibe sombra parcial en algunos meses (abril-agosto). Otras consideraciones como confort e integración arquitectónica han sido también consideradas para el entubado y cableado necesario (ver Figura 6).

La selección de los paneles en el balcón dependió del largo disponible, el cual fue $2.80 \mathrm{~m}$ de largo. Se eligieron 3 paneles de $65 \mathrm{Wp}$ para lograr una superficie neta de celdas de casi $1 \mathrm{~m}^{2}$, similar a lo sugerido en estudios sobre construcción sostenible en el Perú (Miranda et. al, 2015) con las características según la Tabla 1.

Tabla 1. Características Físicas de la Instalación

\begin{tabular}{r|c|c|r}
\hline Potencia: & 3 paneles $65 \mathrm{Wp} \mathrm{c} / \mathrm{u}$ & Área total paneles: & $1.30 \mathrm{~m}^{2}$ \\
Tipo: & Monocristalino & Área neta de celdas: & $1.08 \mathrm{~m}^{2}$ \\
Área panel: & $0.434 \mathrm{~m}^{2} \mathrm{c} / \mathrm{u}$ & Área departamento & $81.80 \mathrm{~m}^{2}$ \\
\hline
\end{tabular}

Fuente: Tomado de Valdivia, R. (2016) 
Las instalaciones están conformadas por:

1) Dos paneles a los extremos del balcón destinados a inyección a la red de la vivienda (micro-inversor) logrando 130Wp. Esta carga no es considerable por lo que representa una inyección pequeña solo para consumo interno sin proporcionar a la red exterior.

2) Un panel central para un sistema de acumulación de $65 \mathrm{Wp}$ (controlador, batería e inversor), el cual abastecería solo una zona interna conformada por un televisor de 26", decodificador, y 10 dicroicos de bajo consumo led de uso esporádico.

3) Finalmente, dos lámparas solares independientes para luz de velador en las ventanas de dos dormitorios (Figura 7), para ser usadas a partir de las 6 p.m., reduciendo el uso de las luminarias conectadas a la red. Este procedimiento fue más aprovechado desde 2019 y obligatorio desde 2020.

El objetivo fundamental de estas instalaciones no es generar toda la electricidad de la vivienda sino una reducción del consumo eléctrico.

Figura 7. Ubicación de Panel en Ventana para Lámpara Solar Interior

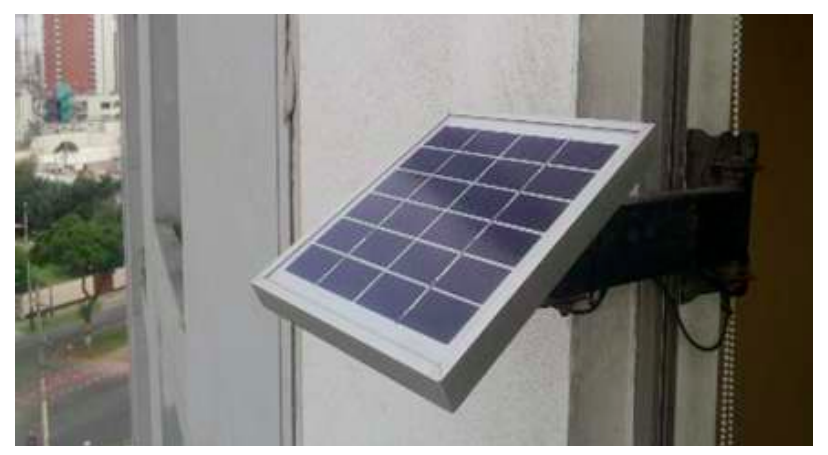

Fuente: Tomado de Valdivia, R. (2019)
Monitoreo de Consumo, Ahorro de Emisiones y Ahorros Económicos

Para el monitoreo, evolución de consumos y costos, se recolectaron los recibos mensuales de electricidad del departamento desde 2008. El promedio de consumo y costo de los dos años antes de la instalación (2015-2016) fue de $208.60 \mathrm{~kW} / \mathrm{h}$ y S/. 122.21 al mes, y constituyen una línea de base comparativa. El principal periodo para los cálculos es el tiempo posterior a la instalación (2017-2020).

Para el balance de emisiones ahorradas por ahorros de electricidad, se recurrió a dos fuentes nacionales: Informe de Identificación de gases de efecto Invernadero, realizado por el Proyecto IKI-PNUMA (MINAM y PUCP, 2017), así como la Guía para elaborar Medidas de Mitigación para Municipalidades Peruanas (Foro Ciudades para la Vida, 2020). Para la matriz energética eléctrica, se ha considerado al año 2018, en que la generación de energía hidráulica y energía térmica (gas natural) generada en la región centro fueron de $69 \%$ y $31 \%$, respectivamente (MEM, 2019).

Para determinar valores confiables de costos y retornos, se ha considerado el incremento del costo de la electricidad tomando como referencia los informes técnicos anuales del Instituto Nacional de Estadística (INEI) desde el 2012. Como se sabe, el costo de electricidad varía en el tiempo y depende de muchos factores. Un promedio del porcentaje de incremento se ha establecido para los años luego de la instalación, 2017 en adelante.

\section{RESULTADOS}

Se demuestra que -incluso con un panorama adverso - con hábitos de ahorro es posible la introducción de energía fotovoltaica de escala pequeña y de aporte parcial con retribuciones ambientales y económicas considerables y mejoradas respecto a 
instalaciones grandes. A nivel de emisiones, se demuestra un aporte significativo, y a nivel económico, se ha reducido el tiempo de recuperación de la inversión.

\section{Comparación de Consumos Eléctricos Anuales}

En base al promedio de dos años previos a la instalación (2015-2016), el consumo al inicio del año era cercano a $150 \mathrm{~kW} / \mathrm{mes}$, mientras que para el invierno se incrementó hasta $300 \mathrm{~kW} / \mathrm{mes}$, prácticamente el doble. Los tres primeros años de instalación fotovoltaica (2017, 2018 y 2019) evidencian una disminución notable del consumo respecto al promedio establecido. A lo largo de los años de instalación, el consumo se redujo logrando aplanar la curva.

El 2020 fue singular por la pandemia del COVID-19. Se incrementó la permanencia en el hogar, pero también permitió un control constante del consumo. Como consecuencia, se evidenció un récord de bajo consumo, menor al límite FOSE de 100kW/mes (Figura 8). La causa de este desempeño se atribuye a la experiencia ganada durante años previos en el ahorro, la acumulación para uso nocturno a partir de las $18.00 \mathrm{~h}$ y el control constante, lo cual posibilitó una reducción considerable del consumo eléctrico a nivel anual (Figura 9):

Figura 8. Comparación del Consumo Promedio Previo (2015-2016) y Luego de la Instalación (2017-2020)

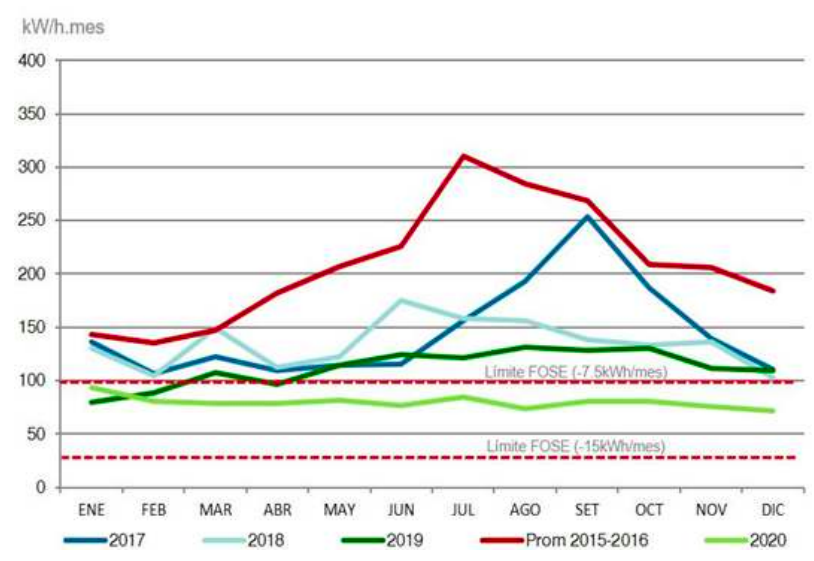

Nota: Elaboración con base en recibos eléctricos 2015-2020 del departamento.
Figura 9. Evolución de Consumos Anuales de Electricidad 2008-2020

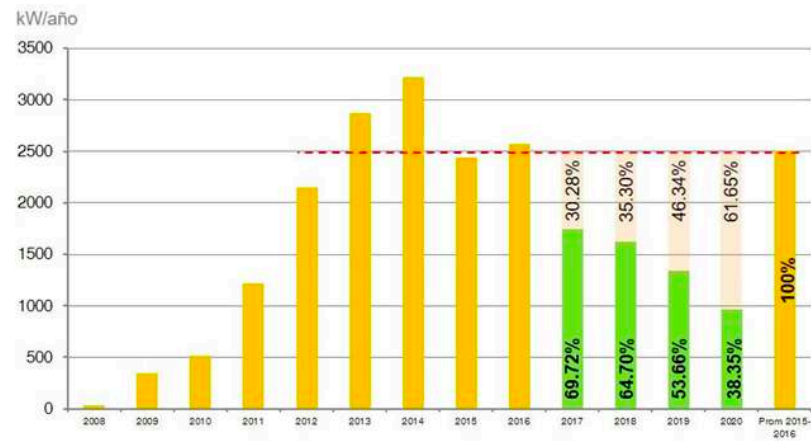

Nota: Elaboración con base en recibos eléctricos 2008-2020 del departamento.

- El 2017, se utilizó el sistema sin mucho rigor, confiando en lo generado por el sistema de inyección, usando poco el sistema de acumulación. El mayor consumo se dio en invierno por el uso de la terma y un radiador en un dormitorio. La reducción de consumo fue de $30.28 \%$ con respecto al promedio previo 2015-2016.

- El 2018, se cambiaron las luminarias ahorradoras a luminarias tipo led. Se reemplazó la terma eléctrica por otra con indicador de temperatura, lo cual permitió un mejor control del consumo, y el logro de una reducción ligera del consumo a $35.30 \%$ respecto al promedio 2015-2016.

- En 2019, se impusieron mayores hábitos ahorradores: se evitó la iluminación durante la noche (sobre todo de los ambientes no utilizados), se utilizaron lámparas solares y el sistema acumulador a partir de las $18.00 \mathrm{~h}$ en el que la zona a ser alimentada por el sistema es usada de manera constante. Durante el verano, la batería tenía una autonomía de hasta 4 horas, pero durante el invierno se usó dejando un día para 2 horas de autonomía. La reducción de consumo fue $46.34 \%$ del promedio 2015-2016.

- Para 2020, los residentes mantuvieron actividades similares hasta el inicio del 
confinamiento (marzo), luego del cual permanecieron con actividades de teletrabajo y telestudio usando computadoras (2 laptops) así como televisión, DVD, y radio durante momentos libres; además de instalaciones de uso constante pero de bajo consumo (celulares, router, decodificador de TV, teléfono inalámbrico) e instalaciones de funcionamiento eventual básico para el hogar (terma, refrigeradora, lavadora, y horno microondas). Se conservaron los hábitos ahorradores del 2019. De esta manera, el consumo de 2020 establece una reducción significativa de $60.20 \%$ respecto al promedio 2015-2016; logro considerable en condiciones no ideales de radiación, orientación, ubicación y normatividad para el uso fotovoltaico. Con las condiciones de "nueva normalidad" el departamento ha logrado un promedio de consumo eléctrico de $79.8 \mathrm{~kW} / \mathrm{h}$ al mes al finalizar el 2020. Esto ha ayudado al logro del subsidio FOSE todos los meses, al evitar un consumo mensual mayor a $100 \mathrm{kWh} / \mathrm{mes}$ mejorando el retorno económico.

En el período total de instalación fotovoltaica (2017-2020), se ahorró 4347.40 kW según el promedio establecido previo a la instalación.

\section{DISCUSIÓN}

\section{Comparación y Estimaciones de $\mathrm{CO} 2$}

Para el cálculo de emisiones evitadas, se usó una lógica de conversión proporcional. Según la Guía para elaborar Medidas de Mitigación para Municipalidades Peruanas (Foro Ciudades para la Vida, 2020) al generar 1 $k W$ de electricidad por hidroeléctricas se emiten $6.8 \mathrm{kgCO}$ eq y al generar $1 \mathrm{~kW}$ de electricidad por centrales térmicas a gas se emiten 415 kgCO2eq. Tomando en cuenta su participación proporcional en la matriz de 2018 , se han emitido por cada $\mathrm{kW}$ de electricidad 133.34 kgCO2eq.

De esta manera, se habrían ahorrado 579.69 tnCO2eq (Figura 10) durante el periodo 2017-2020. Esta estimación de emisiones evitadas establece una referencia de análisis ambiental en departamentos que evidencian la reducción de sus consumos eléctricos, de modo que se pudo aplicar programas de declaración de emisiones como NABERS (National Australian Building Environment Rating, 2020), trabajando con la facturación de energía y reportándose el consumo de viviendas y oficinas entre otros.

Figura 10. Evolución de Emisiones Evitadas en kgC02eq (2016-2020)

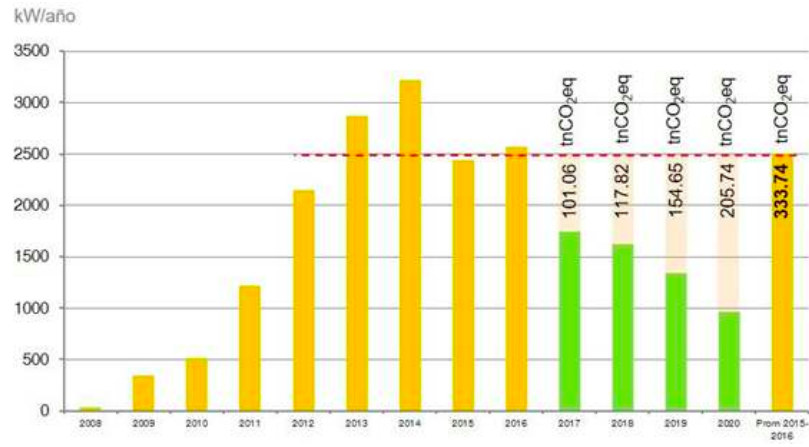

Nota: Elaboración con base en recibos eléctricos 2008-2020 del departamento y datos de la Guía para elaborar Medidas de Mitigación para Municipalidades Peruanas (Foro Ciudades para la Vida, 2020)

\section{Comparación de Costos y Retornos}

De acuerdo a un análisis longitudinal (INEI, 2012-2019), el promedio de incremento anual entre 2017 y 2020 fue de $4.23 \%$, y permite estimar los ahorros en el caso de haber seguido consumiendo como en los 2 años previos a la instalación (2015 y 2016). Según este procedimiento, el ahorro acumulado es de $\mathrm{S} /$. 2,726.31 (Figura 11).

Es imposible determinar la evolución a futuro del incremento de la electricidad, pero se estima que continuará a un ritmo similar. Por ello, el retorno se deduce en función de los años 
transcurridos para estimar ahorros reales y los ahorros futuros pueden ser establecidos de acuerdo a la tendencia y posibles escenarios.

Figura 11. Comparación de Costos Anuales del Consumo de Electricidad del Departamento y Ahorros Generados por el Sistema Fotovoltaico

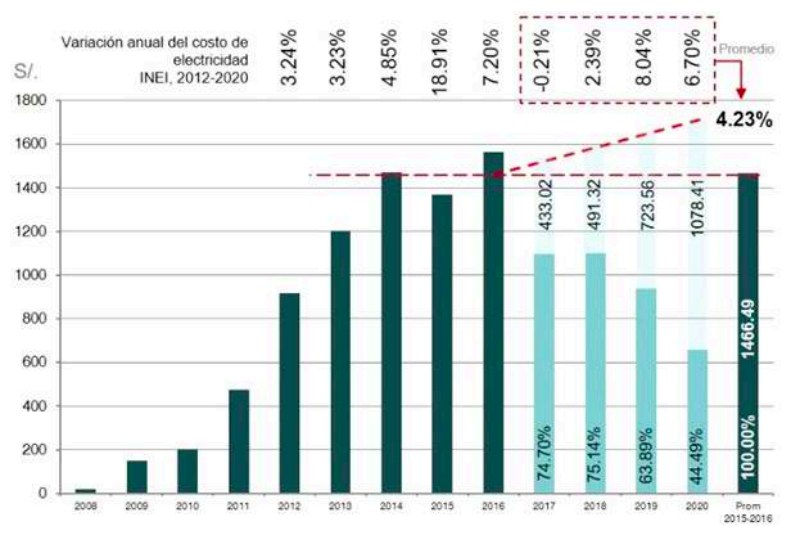

Nota: Elaboración en base a recibos eléctricos 2008-2020 del departamento y porcentaje de variación anual de electricidad según INEI 2012 a 2020

- El costo de electricidad en 2017 fue de $74.70 \%$ del promedio de los 3 años previos a la instalación, logrando ahorros notables (25.30\%).

- El costo de electricidad en 2018 solo fue de $75.14 \%$ del promedio de los años previos a la instalación, obteniendo un ahorro similar respecto al promedio 2015-2016 (24.86\%).
- El costo de electricidad del 2019 llegó a reducirse al $63.89 \%$ de los años previos a la instalación obteniendo un ahorro muy significativo (36.11\%).

- El costo del año 2020 es el 44.71\% del promedio de los años previos a la instalación (2015-2016), marcando un ahorro muy considerable (55.51\%).

Como muestra la Tabla 2 , si el costo de la instalación mixta fue de $\mathrm{S} / 5,250$ (finales de 2016), en un 'modo de aprendizaje' se compara el escenario de costo eléctrico anual en aumento de $4.23 \%$ así como el consumo convencional previo a la instalación, seguidamente con el escenario de ahorros registrados con la instalación y hábitos aprendidos de 2017 a 2020 (amarillo) y proyectados a 2023 (naranja). Luego, se puede deducir un retorno en 6.2 años considerando los ahorros acumulados proyectados (verde). Sin embargo, en un 'modo mejorado' comparando un escenario convencional (aumento de la tarifa anual y consumo despreocupado) pero con una instalación y hábitos ahorradores, considerando la experiencia ganada al 2020 (color amarillo), y si se mantuvieran las condiciones con los ahorros posibles a futuro (naranja), con el acumulado obtenido (verde), el retorno podría llegar a ser en 4.5 años (Tabla 2).

Tabla 2. Retornos Anuales Registrados (Amarillo), Acumulados Posibles (verde) y Proyectados (Naranja) en S/.

\begin{tabular}{|c|c|c|c|c|c|c|c|}
\hline Modo Aprendizaje & $\begin{array}{c}2017 \\
1\end{array}$ & $\begin{array}{c}2018 \\
2\end{array}$ & $\begin{array}{c}2019 \\
3\end{array}$ & $\begin{array}{c}2020 \\
4\end{array}$ & $\begin{array}{c}2021 \\
5\end{array}$ & $\begin{array}{c}2022 \\
6\end{array}$ & $\begin{array}{c}2023 \\
7\end{array}$ \\
\hline Costo eléctrico anual en S/ & 1516.35 & 1567.90 & 1621.21 & 1676.33 & 1733.33 & $3 \mid 1792.26$ & 1853.20 \\
\hline Ahorros Registrados en S/ & 433.02 & 491.32 & 723.56 & 1078.413 & 1124.02 & 21171.57 & 1221.13 \\
\hline Retornos acumulados & \multicolumn{4}{|c|}{2726.31} & 3850.33 & 5266.13 & 36243.03 \\
\hline Tiempo de retorno & \multicolumn{6}{|c|}{6.2 años } & \\
\hline Modo Mejorado & $\begin{array}{c}2020 \\
1\end{array}$ & \begin{tabular}{|c|}
2021 \\
2
\end{tabular} & \begin{tabular}{|c|}
2022 \\
3
\end{tabular} & \begin{tabular}{|c|}
2023 \\
4
\end{tabular} & $\begin{array}{c}2024 \\
5\end{array}$ & \begin{tabular}{|c|c|}
2025 \\
6
\end{tabular} & $\begin{array}{c}2026 \\
7\end{array}$ \\
\hline Costo eléctrico anual en S/ & 1730.81 & 1804.02 & 1880.33 & 1959.87 & 2042.77 & $7 \quad 2129.18$ & 2219.24 \\
\hline Ahorros posibles & 1078.413 & 1124.02 & 1171.57 & 1221.13 & 1272.78 & $8 \mid 1326.62$ & 2382.74 \\
\hline Posibles retornos acumulados S/ & \multicolumn{5}{|c|}{5231.52} & \multirow{2}{*}{\multicolumn{2}{|c|}{$\begin{array}{l}\text { Retribuciones futuras } \\
\longrightarrow\end{array}$}} \\
\hline Tiempo de retorno & & & 5 años & & & & \\
\hline
\end{tabular}


En un supuesto caso de retribuciones con la Ley de Generación Distribuida, el mecanismo Net Metering tendría las tasas de retorno más elevadas, y dependiendo de la potencia en kWp y la zona de instalación en el país, puede recuperar su inversión entre 6 y 7 años en promedio. Mientras que para el caso del Net Billing, dependiendo de la potencia en kWp y la zona de instalación en el país, puede recuperar su inversión entre 8 y 11 años.

Cabe señalar, que, si el usuario efectúa una inversión sin obtener alguna compensación por la energía que inyecta (excedentes) a la red eléctrica sólo podría tener una tasa de descuento de su inversión que oscila entre 1 y $3 \%$ recuperando su inversión aproximadamente en 21 años, lo cual no representa incentivo alguno para el cliente regulado, siendo ello la situación actual de la generación distribuida en el Perú (Palacios et. al, 2020, pp. 65-66)

\section{CONCLUSIONES}

- Los ahorros en el consumo (energía y costos) de instalaciones mixtas son posibles, pero deben estar acompañados de hábitos ahorradores (control) para ser efectivos. Ello teniendo en cuenta el contexto desfavorable: radiación (variable en Lima), sitio (sin acceso a techo), orientación (diferente a la recomendada), dimensión (solo $1 \mathrm{~m}^{2}$ de área fotovoltaica), ausencia de incentivos (sin netmettering o netbilling como mecanismo de retribución), un mercado cautivo a nivel de electricidad (tarifas reguladas y en incremento) así como mantenimiento (limpieza en altura, etc.).

La "nueva normalidad" del confinamiento por la pandemia (trabajo y estudio en casa) favorece el uso de sistemas fotovoltaicos para viviendas de departamentos, incluso de aquellas sin acceso a techo. Sin embargo, los hábitos de ahorro desarrollados a partir de la instalación solar, así como los hábitos y el uso de equipos eficientes deben ser materia de estudios a profundidad en adelante.

- Con sistemas mixtos de producción fotovoltaica de dimensiones pequeñas e intermedias, se puede recuperar la inversión más rápido (6.2 años con lo experimentado y 4.5 años en caso de una conducta encaminada al ahorro desde el inicio). Mientras que con la generación distribuida: netmettering o netbilling (con 6.9 a 10.6 años, respectivamente) aún inaplicada en el Perú y añoradas durante más de una década, se requerirá de un mayor tiempo para el retorno. Los sistemas mixtos de baja producción eléctrica y complementarios al sistema convencional permitirían apostar hacia una microgeneración individual inducida por las circunstancias desfavorables del mercado eléctrico peruano.

- En el caso de Lima, para los sistemas de acumulación fotovoltaica, no se requiere energizar toda la vivienda, sino solo parte de ella o los consumos más viables y adaptables (por ej. iluminación y electrodomésticos de bajo consumo, pero constantes), aprovechando las oportunidades disponibles de radiación de la vivienda.

- El objetivo de instalaciones solares residenciales con las condiciones actuales debería centrarse en llegar a los subsidios del FOSE y reducir el consumo en hora punta. El reto es lograr consumos menores a $100 \mathrm{kWh} /$ mes o incluso menores a $30 \mathrm{kWh} / \mathrm{mes}$ logrando el subsidio FOSE del $7.5 \mathrm{kWh} / \mathrm{mes}$ o del 15 $\mathrm{kWh} / \mathrm{mes}$, respectivamente.

- El uso de paneles solares en balcones significa un territorio a explorar para adaptar instalaciones de apoyo parcial. La diversidad de balcones, sus orientaciones y dimensiones son variables para mayores estudios a futuro en 
relación a la oferta de paneles y el potencial de captación energética. Innovaciones -como la patente 002030-2018/DIN "Soporte colgante para paneles fotovoltaicos en balcones" y otras patentes en curso, como la solicitud de patente 001910-2020/DIN "Soporte colgante para paneles fotovoltaicos con movimiento pendular y giro en su eje vertical" - pueden ser tomadas en cuenta para diseños de departamentos, ayudando a la adaptación de estos espacios, lo cual constituye una herramienta de gran utilidad para programas de descarbonización, mitigación y adaptación; también dichos diseños podrían ser incluidos en programas de viviendas sostenibles para recibir subsidios.

- El monitoreo de las facturaciones mensuales y balances de tipo anual posibilita determinar ahorros energéticos, económicos y de emisiones que avalan este tipo de instalación para ser incluida en programas de vivienda con la finalidad de optar a financiamientos de reducción real de emisiones para adaptación y mitigación (por ej. SREP [Programa de aumento del Aprovechamiento de Fuentes Renovables] en países de bajos ingresos, etc.), o en programas de construcción sostenible (por ej. bono verde MiVivienda), o procesos normativos (por ej. Código Técnico de Construcción Sostenible) e incluso en sistemas de registro nacional/municipal en localidades que deseen monitorear y reducir emisiones (por ej. NABERS). De esta manera, se favorece el incremento de los beneficios económicos y oportunidades para propietarios o usuarios de energía solar, considerándolos más como "prosumidores" ${ }^{8}$ que simples "consumidores".

\section{REFERENCIAS BIBLIOGRÁFICAS}

Alianza Clima y Desarrollo. (30 de Marzo de $2020)$. https: / / cdkn.orgl. https://cdkn.org/2020/03/opinion-la-ruta-delos-fondos-climaticos-internacionales-enamerica-latina/?loclang=es es

Arias, D. (2017). Sistemas NetMetering y NetBilling. Universidad Tecnológica Centroamericana.

Censolar. (1993). Valores medios de irradiación solar sobre el suelo horizontal. Sevilla Promotora General de Estudios.

Coronado, J. (10 de diciembnre de 2020). Gobernanza actual, principales brechas, obstáculos y desafíos para el acceso a la vivienda y el uso de energía en asentamiento informales en la ciudad de Lima. Proyecto GEMDev. (L. Miranda, M. Verdiere, y R. H. Valdivia-Sisniegas, Entrevistadores).

EIA. (2016). Indicadores de Eficiencia Energética. Fundamentos estadísticos. Energy Data Centre EIA.

Foro Ciudades para la Vida. (2020). Guía para elaborar Medidas de Mitigación para Municipalidades Peruanas. IUC.

Gutiérrez, C. (14 de junio de 2019). Tarifas eléctricas: Distorsión de precios en Perú. Proyecto Alcalá. El reclamo a Viscarra. htt $\mathrm{p}: / / \mathrm{min}$ ing press.com/: http://miningpress.com/322347/tarifaselectricas-distorsion-de-precios-en-peruproyecto-alcala-el-reclamo-a-vizcarra

Humpire, D. (2017). Análisis Comparativo de los Mecanismos Net Metering y Net Billing para Generación Distribuida Fotovoltaica Residencial en el Mercado Eléctrico Peruano. XXIV Simposio Peruano de Energía Solar y del Ambiente (SPES). Universidad Nacional de Ingeniería, Maestría en Energética.

\footnotetext{
${ }^{8}$ Del inglés 'prosumer', fusión original de las palabras 'productor' y 'consumidor'. Alguien que produce lo que consume.
} 
INEI. (2012-2019). Variación de indicadores de precios de la economía. INEI. www.inei.gob.pe

"Tarifa" de electricidad se encareció $10 \%$ durante la pandemia. (10 de abril de 2021). La República.

Matos, M., y Vargas, E. (2019). La generación distribuida como forma de acceder al autoconsumo eléctrico a pequeña escala. (Santivañez Abogados, Ed.) Revista Peruana de Energía, 7, 32-58.

https://www.santivanez.com.pe/rpdenergia7/

Meier, P., Tuntivate, V., Barnes, D. F., Bogach, S. V., y Farchy, D. (2010). PERÚ Encuesta Nacional de Consumo de Energía a Hogares en el Ámbito Rural. Unidad de Energía Departamento de Desarrollo Sostenible Región de América Latina yel Caribe Banco Mundial.

MEM. (2017). Uso eficiente de la energía. Guía metodológica para docentes de Inicial-Primaria. Ministerio de Energía y Minas del Perú.

MEM. (2019). Principales indicadores del sector eléctrico a nivel nacional. Ministerio de Energía y Minas del Perú. Dirección de Estudios y Promoción Eléctrica.

MINAM y PUCP. (2017). Proyecto IKI-PNUMA. Informe de identificación de gases de efecto invernadero (GEIS) en subsectores del Perú y análisis del potencial de mitigación. PUCP, ONU, OyFP, Ministerio Federal de Medio Ambiente, Protección de la Naturaleza, Obras Públicas y Seguridad Nuclear de Alemania.

Miranda, L., Neira , E., Torres, R., Valdivia, R. (2015). Perú Hacia la Construcción Sostenibles en Escenarios del Cambio Climático. Universidad Ricardo Palma.

MVCS. (2015). Código Técnico de Construcción Sostenible. Ministerio de Vivienda Construcción y Saneamiento.
National Australian Building Environment Rating, S. (10 de enero de 2020). https://www.nabers.gov.au/.

Okumura, P. A. (2015). El mercado mayorista de electricidad en el Perú. Themis. Revista de Derecho(68), 261-277.

http://revistas.pucp.edu.pe/index.php/themis/ article/view/15598

Osinergmin. (2017). Informe de resultados: Encuesta residencial de uso y consumo de energía-ERCUE 2014-2015. GPAE.

Osinermin. (2017). Industria de la Electricidad en el Perú. 25 años de aporte al crecimiento económico. Organismo Supervisor de la Inversión en Energía y Minería.

Osinermin. (2018). Reporte de Análisis económico sectorial Sector Electricidad. Generación distribuida: marco comparado para Perú, Chile, Colombia y México. Gerencia de Políticas y Análisis Económicos.

Palacios, David; Rojas, Rubén; Ramírez, Edwin. (2020). Aspectos Regulatorios a considerar en la implementación de la microgeneración distribuida residencial fotovoltaica en el muercado eléctrico peruano. Universidad Peruana de Ciencias.

Periferia y World Wild Fundation. (2018). Ciudades del Perú. Primer Reporte Nacional de Indicadores Urbanos 2018. Con un enfoque de sostenibilidad y resiliencia. Periferia y World Wild Fundation.

Ramos, E. (2020). La generación distribuida: El camino hacia la producción descentralizada de electricidad y pautas para su reglamentación. Forseti Revisgta de Derecho, 8(11), 07-35.

Revista Energía.pe Electricidad e Hidrocarburos. (26 de noviembre de 2019). 
https://revistaenergia.pe/peru-demora-elnuevo-reglamento-para-incentivar-generaciondistribuida/

Sciutto, W. (2019). Impactos de la generación distribuida para las empresas distribuidoras en el contexto actual. (Santivañez Abogados, Ed.) Revista Peruana de Energía, 7, 21-34. https://www.santivanez.com.pe/rpdenergia7/

Valdivia-Sisniegas, R. (2017). Balcones Resilientes. Introduciendo seguridad hídrica, energética y alimentaria en viviendas. Arquitextos(32), 91-102.
Valdivia-Sisniegas, R. (19 de octubre de 2018). Perú Patente no 002030-2018/DIN. https://patents.google.com/patent/PE2019156 7Z/en?oq=patent+PE+pe20191567z

Valdivia-Sisniegas, R. (2 de diciembre de 2020). Perú Patente no Exp:001910-2020/DIN.

Valdivia-Sisniegas, R. H. (2015). Consumos de Electricidad y Agua en viviendas de estudiantes en Lima 2011-2014. [Sin publicar]. 